LTH-714

hep-th/0610118

\title{
Minimal Standard Heterotic String Models
}

\author{
Alon E. Faraggi*, Elisa Manno ${ }^{\dagger}$ and Cristina Timirgaziu ${ }^{\S}$ \\ Department of Mathematical Sciences, University of Liverpool, Liverpool L69 7ZL
}

\begin{abstract}
Three generation heterotic-string vacua in the free fermionic formulation gave rise to models with solely the MSSM states in the observable Standard Model charged sector. The relation of these models to $Z_{2} \times Z_{2}$ orbifold compactifications dictates that they produce three pairs of untwisted Higgs multiplets. The reduction to one pair relies on the analysis of supersymmetric flat directions, that give superheavy mass to the dispensable Higgs states. We explore the removal of the extra Higgs representations by using the free fermion boundary conditions and hence directly at the string level, rather than in the effective low energy field theory. We present a general mechanism that achieves this reduction by using asymmetric boundary conditions between the left- and right-moving internal fermions. We incorporate this mechanism in explicit string models containing three twisted generations and a single untwisted Higgs doublet pair. We further demonstrate that an additional effect of the asymmetric boundary conditions is to substantially reduce the supersymmetric moduli space.
\end{abstract}

\footnotetext{
*E-mail address: faraggi@amtp.liv.ac.uk

$\dagger$ E-mail address: Elisa.Manno@liv.ac.uk

$\S$ E-mail address: timirgaz@amtp.liv.ac.uk
} 


\section{Introduction}

String theory provides a viable framework to probe the unification of gravity and the gauge interactions. Preservation of classical symmetries in the quantised string fixes the number of world-sheet degrees of freedom required for internal consistency. These can be taken as bosonic degrees of freedom and interpreted as additional dimensions, beyond the four space-time, or as an internal two dimensional conformal field theory on the string world-sheet. String states are obtained by acting on the nondegenerate vacuum with bosonic and fermionic excitations and give rise to the matter and interaction states that constitute the experimentally observed spectrum. String theory in four dimensions gives rise to a multitude of consistent vacuum solutions and the selection of the ones relevant for experimental investigation is one of the perplexing issues in string theory.

On the other hand, the data extracted from collider and other contemporary experiments highlights the Standard Particle Model as the correct accounting of all the observed data. Furthermore, the particle physics data is compatible with the hypothesis that the renormalisable Standard Model remains unaltered up to a large energy scale and that the particle spectrum is embedded in a Grand Unified Theory (GUT) [1]. Most appealing in this context is $\mathrm{SO}(10)$ unification, in which each Standard Model generation is embedded in a single 16 spinorial representation. The hypothesis of unification is further supported by the observed logarithmic evolution of the Standard Model parameters, by the suggestive compatibility of coupling unification with the low energy data and by the suppression of proton decay mediating operators and left-handed neutrino masses. However, as many of the Standard Model variables are mere parameters in the context of GUTs, their origin is not explained in the framework of point quantum field theories. Therefore, elucidating further the properties of the Standard Model spectrum, such as the existence of flavour, necessitates the unification of the Standard Particle Model with gravity. String theory therefore provides a unique framework to explore how the Standard Model parameters may arise from such unification.

Toward this end and lacking a mechanism that dynamically selects a unique string vacuum in four dimensions, the Standard Model data is used to single out phenomenologically viable string vacua. Maintaining the $\mathrm{SO}(10)$ embedding of the Standard Model spectrum necessitates that we study compactifications of the heterotic string [2], as spinorial $S O(10)$ representations are obtained in the heterotic string [2], but not in the other perturbative string theories. The compatibility of the low energy data with gauge coupling unification indicates that the observable gauge group below the string scale should be $S U(3)_{C} \times S U(2)_{L} \times U(1)_{Y}$ and that the $S U(3)_{C} \times S U(2)_{L} \times U(1)_{Y}$-charged spectrum should consist solely of the Minimal Supersymmetric Standard Model spectrum. Indeed, over the past year there has been a resurgence of interest in the construction of phenomenologically viable heterotic string vacua [3]. We therefore recall that the semi-realistic models in the free fermionic formulation [4-11] produced in the past solutions in which the only 
Standard Model charged states are the MSSM states [11]. It is therefore an opportune moment to revisit these models and to examine some of their properties.

One of the intriguing successes of the free fermionic standard-like models has been the successful prediction of the top quark mass several years prior to its experimental observation [9]. Furthermore, the models offered an explanation why only the top quark mass is characterised by the electroweak scale, whereas the masses of the lighter quarks and leptons are suppressed. The reason being that only the top quark Yukawa coupling is obtained at the cubic level of the superpotential, whereas the Yukawa couplings of the lighter quarks and leptons are obtained from nonrenormalizable terms which are suppressed relative to the leading order term. In the free fermionic quasi-realistic standard-like models the three generations arise from the three twisted sectors, whereas the Higgs doublets, to which they couple in leading order, arise from the untwisted sector. At leading order each twisted generation couples to a separate pair of untwisted Higgs doublets. Analysis of supersymmetric flat directions implied that at low energies only one pair of Higgs doublets remains light and other Higgs doublets obtain heavy mass from VEVs of Standard Model singlet fields. Hence, in the low energy effective field theory, only the coupling of the twisted generation that couples to the light Higgs remains at leading order. The consequence is that only the top quark mass is obtained at leading order, whereas the masses of the remaining quarks and leptons are obtained at subleading orders. Evolution of the calculated Yukawa couplings from the string to electroweak scale (and using the low energy data for the bottom quark and electroweak vector bosons) then yields a prediction for the top quark mass. An additional constraint on the analysis is that the gauge coupling unification at the high scale is compatible with the low energy data.

The analysis of the top quark mass therefore relies on the analysis of supersymmetric flat directions and the decoupling of the additional untwisted electroweak Higgs doublets, that couple to the twisted generations at leading order. In this paper we examine whether an alternative construction is possible. Our aim is to construct models in which only one pair of untwisted Higgs doublets remains in the massless spectrum after application of the Generalised GSO (GGSO) projections. Therefore, the massless string spectrum contains a single electroweak Higgs doublet pair, without relying on analysis of supersymmetric flat direction in the effective low energy field theory. Consequently, at leading order only the top quark couples to the electroweak Higgs doublet, and therefore only its mass is intrinsically associated with the electroweak scale.

Our paper is organised as follows. In section 2 we review the structure of the minimal standard heterotic string models in the free fermionic formulation. In section 2.2 we describe in general the mechanism that projects the additional untwisted Higgs doublets, in terms of the free fermion boundary condition basis vectors. In section 3 we present an explicit string model which contains a single untwisted Higgs doublet pair and present its full massless spectrum as well as the full cubic level superpotential. Section 3 also contains a discussion of the flat directions. Section 4 concludes the paper. 


\section{Minimal Standard Heterotic String Models}

In this section we briefly review the construction and structure of the free fermionic standard like models. The notation and further details of the construction of these models are given elsewhere $[5,8,10-12]$. In the free fermionic formulation of the heterotic string in four dimensions [13] all the world-sheet degrees of freedom, required to cancel the conformal anomaly, are represented in terms of free fermions propagating on the string world-sheet. In the light-cone gauge the world-sheet field content consists of two transverse left- and right-moving space-time coordinate bosons, $X_{1,2}^{\mu}$ and $\bar{X}_{1,2}^{\mu}$, and their left-moving fermionic superpartners $\psi_{1,2}^{\mu}$, and additional 62 purely internal Majorana-Weyl fermions, of which 18 are left-moving, and 44 are right-moving. The models are constructed by specifying the phases picked by the world-sheet fermions when transported along the torus non-contractible loops. Each model corresponds to a particular choice of fermion phases consistent with modular invariance and is generated by a set of basis vectors describing the transformation properties of the 64 world-sheet fermions. The physical spectrum is obtained by applying the generalised GSO projections. The low energy effective field theory is obtained by $\mathrm{S}$-matrix elements between external states [14].

The boundary condition basis defining a typical realistic free fermionic heterotic string models is constructed in two stages. The first stage consists of the NAHE set, which is a set of five boundary condition basis vectors, $\left\{\mathbf{1}, S, b_{1}, b_{2}, b_{3}\right\}[10,15]$. The gauge group after imposing the GSO projections induced by the NAHE set is $S O(10) \times S O(6)^{3} \times E_{8}$ with $N=1$ supersymmetry. The NAHE set divides the internal world-sheet fermions in the following way: $\bar{\phi}^{1, \cdots, 8}$ generate the hidden $E_{8}$ gauge group, $\bar{\psi}^{1, \cdots, 5}$ generate the $S O(10)$ gauge group, and $\left\{\bar{y}^{3, \cdots, 6}, \bar{\eta}^{1}\right\},\left\{\bar{y}^{1}, \bar{y}^{2}, \bar{\omega}^{5}, \bar{\omega}^{6}, \bar{\eta}^{2}\right\}$, $\left\{\bar{\omega}^{1, \cdots, 4}, \bar{\eta}^{3}\right\}$ generate the three horizontal $S O(6)$ symmetries. The left-moving $\{y, \omega\}$ states are divided to $\left\{y^{3, \cdots, 6}\right\},\left\{y^{1}, y^{2}, \omega^{5}, \omega^{6}\right\},\left\{\omega^{1, \cdots, 4}\right\}$, while $\chi^{12}, \chi^{34}, \chi^{56}$ generate the left-moving $N=2$ world-sheet supersymmetry. At the level of the NAHE set the sectors $b_{1}, b_{2}$ and $b_{3}$ produce 48 multiplets, 16 from each, in the 16 representation of $S O(10)$, that are singlets of the hidden $E_{8}$ gauge group and transform under the horizontal $S O(6)_{j}(j=1,2,3)$ symmetries. The untwisted sector produces states in the 10 vectorial representation of $S O(10)$ that can produce electroweak Higgs doublets. At this stage we anticipate that the $S O(10)$ group produces the Standard Model gauge group factors, and that the $16 \cdot 16 \cdot 10$ can produce the Standard Model fermion mass terms. This structure is common to all the realistic free fermionic models that we consider here.

The second stage of the basis construction consists of adding to the NAHE set three additional boundary condition basis vectors. These additional basis vectors reduce the number of generations to three chiral generations, one from each of the sectors $b_{1}, b_{2}$ and $b_{3}$, and simultaneously break the four dimensional gauge group. The assignment of boundary conditions to $\left\{\bar{\psi}^{1, \cdots, 5}\right\}$ breaks $S O(10)$ to one of its subgroups $S U(5) \times U(1)[4], S O(6) \times S O(4)[7], S U(3) \times S U(2) \times U(1)^{2}[5,8,11], S U(3) \times S U(2)^{2} \times$ $U(1)[12]$ or $S U(4) \times S U(2) \times U(1)$ [16]. Similarly, the hidden $E_{8}$ symmetry is broken 
to one of its subgroups. The flavour $S O(6)^{3}$ symmetries in the NAHE-based models are always broken to flavour $U(1)$ symmetries, as the breaking of these symmetries is correlated with the number of chiral generations. Three such $U(1)_{j}$ symmetries are always obtained in the NAHE based free fermionic models, from the subgroup of the observable $E_{8}$, which is orthogonal to $S O(10)$. These are produced by the world-sheet currents $\bar{\eta} \bar{\eta}^{*}(j=1,2,3)$, which are part of the Cartan sub-algebra of the observable $E_{8}$. Additional unbroken $U(1)$ symmetries, denoted typically by $U(1)_{j}(j=4,5, \ldots)$, arise by pairing two real fermions from the sets $\left\{\bar{y}^{3, \cdots, 6}\right\},\left\{\bar{y}^{1,2}, \bar{\omega}^{5,6}\right\}$ and $\left\{\bar{\omega}^{1, \cdots, 4}\right\}$. The final observable gauge group depends on the number of such pairings. Alternatively, a left-moving real fermion from the sets $\left\{y^{3, \cdots, 6}\right\},\left\{y^{1,2}, \omega^{5,6}\right\}$ and $\left\{\omega^{1, \cdots, 4}\right\}$ may be paired with its respective right-moving real fermion to form an Ising model operator, in which case the rank of the right-moving gauge group is reduced by one. The reduction of untwisted electroweak Higgs doublets crucially depends on the pairings of the left- and right-moving fermions from the set $\{y, \omega \mid \bar{y}, \bar{\omega}\}^{1 \cdots 6}$.

Subsequent to constructing the basis vectors and extracting the massless spectrum, the analysis of the free fermionic models proceeds by calculating the superpotential. The cubic and higher-order terms in the superpotential are obtained by evaluating the correlators

$$
A_{N} \sim\left\langle V_{1}^{f} V_{2}^{f} V_{3}^{b} \cdots V_{N}^{b}\right\rangle
$$

where $V_{i}^{f}\left(V_{i}^{b}\right)$ are the fermionic (scalar) components of the vertex operators, using the rules given in [14]. Generically, correlators of the form (2.1) are of order $\mathcal{O}\left(g^{N-2}\right)$, and hence of progressively higher orders in the weak-coupling limit. Typically, one of the $U(1)$ factors in the free-fermion models is anomalous and generates a FayetIliopoulos term which breaks supersymmetry at the Planck scale [17]. A supersymmetric vacuum is obtained by assigning non-trivial VEVs to a set of Standard Model singlet fields in the massless string spectrum along $F$ and $D$-flat directions. Some of these fields will appear in the nonrenormalizable terms (2.1), leading to effective operators of lower dimension. Their coefficients contain factors of order $\mathcal{V} / M \sim 1 / 10$.

An example of free fermionic standard-like model is given in table 2.2.

\begin{tabular}{|c|c|c|c|c|c|c|c|c|c|}
\hline & $\psi^{\mu}$ & $\chi^{12}$ & $\chi^{34}$ & $\chi^{56}$ & $\bar{\psi}^{1, \ldots, 5}$ & $\bar{\eta}^{1}$ & $\bar{\eta}^{2}$ & $\bar{\eta}^{3}$ & $\bar{\phi}^{1, \ldots, 8}$ \\
\hline$b_{4}$ & 1 & 1 & 0 & 0 & $\begin{array}{lllll}11111 & 1\end{array}$ & 1 & 0 & $\overline{0}$ & $\begin{array}{llllllllll} & 0 & 0 & 0 & 0 & 0 & 0 & 0\end{array}$ \\
\hline$\alpha$ & 1 & 0 & 0 & 1 & 11100 & 1 & 1 & 0 & 11110000 \\
\hline$\beta$ & 1 & 0 & 1 & 0 & 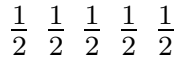 & $\frac{1}{2}$ & $\frac{1}{2}$ & $\frac{1}{2}$ & $\frac{1}{2} 0111 \frac{1}{2} \frac{1}{2} \frac{1}{2} 0$ \\
\hline
\end{tabular}

\begin{tabular}{c|cccc|cccc|cccc} 
& $y^{3} y^{6} y^{4} \bar{y}^{4} y^{5} \bar{y}^{5} \bar{y}^{3} \bar{y}^{6}$ & \multicolumn{2}{|c|}{$y^{1} \omega^{6} y^{2} \bar{y}^{2} \omega^{5} \bar{\omega}^{5} \bar{y}^{1} \bar{\omega}^{6}$} & \multicolumn{4}{c}{$\omega^{1} \omega^{3} \omega^{2} \bar{\omega}^{2} \omega^{4} \bar{\omega}^{4} \bar{\omega}^{1} \bar{\omega}^{3}$} \\
\hline \hline$b_{4}$ & 1 & 0 & 0 & 1 & 0 & 0 & 1 & 0 & 0 & 0 & 1 & 0 \\
$\alpha$ & 0 & 0 & 0 & 1 & 0 & 1 & 0 & 1 & 1 & 0 & 1 & 0 \\
$\beta$ & 0 & 0 & 1 & 1 & 1 & 0 & 0 & 1 & 0 & 1 & 0 & 0
\end{tabular}


The choice of generalised GSO coefficients is:

$$
\begin{aligned}
& c\left(\begin{array}{c}
b_{4} \\
b_{j}, \alpha
\end{array}\right)=-c\left(\begin{array}{c}
b_{4} \\
\mathbf{1}
\end{array}\right)=-c\left(\begin{array}{c}
\alpha \\
\mathbf{1}
\end{array}\right)=c\left(\begin{array}{l}
\alpha \\
b_{j}
\end{array}\right)= \\
& -c\left(\begin{array}{c}
\alpha \\
\beta
\end{array}\right)=c\left(\begin{array}{c}
\beta \\
b_{2}
\end{array}\right)=-c\left(\begin{array}{c}
\beta \\
b_{1}, b_{3}, b_{4}, \beta
\end{array}\right)=-1
\end{aligned}
$$

$(j=1,2,3)$, with the others specified by modular invariance and space-time supersymmetry. The full massless spectrum, charged under the four dimensional gauge group of this model was presented in ref. [5], as well as the full cubic level superpotential. In ref. [6] it was noted that all the exotic fractionally charged states in the model decouple from the effective low energy field theory at the cubic level of the superpotential, provided that the set of Standard Model singlet fields $\bar{\phi}_{4}, \bar{\phi}_{4}^{\prime}, \phi_{4}, \phi_{4}^{\prime}$ obtain a string scale VEV. Supersymmetric flat solutions that incorporate these VEVs were found in ref. [11], given by the VEVs of the set of fields

$$
\left\{\phi_{12}, \phi_{23}, \bar{\phi}_{56}, \phi_{4}, \phi_{4}^{\prime}, \bar{\phi}_{4}, \bar{\phi}_{4}^{\prime}, H_{15}, H_{30}, H_{31}, H_{38}\right\} \text {. }
$$

Additionally it was demonstrated in [11] that in this vacuum solution the only Standard Model charged states that remain massless below the anomalous $U(1)$ scale consist of the states of the minimal supersymmetric standard model. Such solutions are therefore dubbed Minimal Standard Heterotic String Models (MSHSM).

\subsection{Yukawa Selection Mechanism}

At the cubic level of the superpotential the boundary condition basis vectors fix the cubic level Yukawa couplings for the quarks and leptons [9]. These Yukawa couplings are fixed by the vector $\gamma$ which breaks the $S O(10)$ symmetry to $S U(5) \times$ $U(1)$. Each sector $b_{i}$ gives rise to an up-like or down-like cubic level Yukawa coupling. We can define a quantity $\Delta$ in the vector $\gamma$, which measures the difference between the left- and right-moving boundary conditions assigned to the internal fermions from the set $\{y, w \mid \bar{y}, \bar{\omega}\}$ and which are periodic in the vector $b_{i}$,

$$
\Delta_{i}=\mid \gamma_{L}(\text { internal })-\gamma_{R}(\text { internal }) \mid=0,1 \quad(i=1,2,3)
$$

If $\Delta_{i}=0$ then the sector $b_{i}$ gives rise to a down-like Yukawa coupling while the up-type Yukawa coupling vanishes. The opposite occurs if $\Delta_{i}=1$. In the model of table 2.2 the basis vector responsible for the breaking of $S O(10)$ symmetry to $S U(5) \times U(1)$ is $\beta$ and, therefore, we obtain at the cubic level $Q_{1} u_{1} \bar{h}_{1}, L_{1} N_{1} \bar{h}_{1}, Q_{2} d_{2} h_{2}$, $L_{2} e_{2} h_{2}$ and $Q_{3} d_{3} h_{3}, L_{3} e_{3} h_{3}$, irrespective of the choice of GSO projection coefficients. In models that produce $\Delta_{i}=1$ for $i=1,2,3$ the down-quark type cubic-level Yukawa couplings vanish and the models produce only up-quark type Yukawa couplings at the cubic level of the superpotential. Models with these characteristics were presented in refs. $[8,9]$. 


\section{$2.2 \quad$ Higgs Doublet-Triplet Splitting}

The Higgs doublet-triplet splitting operates as follows [18]. The Neveu-Schwarz sector gives rise to three fields in the 10 representation of $S O(10)$. These contain the Higgs electroweak doublets and colour triplets. Each of those is charged with respect to one of the horizontal $U(1)$ symmetries $U(1)_{1,2,3}$. Each one of these multiplets is associated, by the horizontal symmetries, with one of the twisted sectors, $b_{1}, b_{2}$ and $b_{3}$. The doublet-triplet splitting results from the boundary condition basis vectors which break the $S O(10)$ symmetry to $S O(6) \times S O(4)$. We can define a quantity $\Delta_{i}$ in these basis vectors which measures the difference between the boundary conditions assigned to the internal fermions from the set $\{y, w \mid \bar{y}, \bar{\omega}\}$ and which are periodic in the vector $b_{i}$,

$$
\Delta_{i}=\mid \alpha_{L}(\text { internal })-\alpha_{R}(\text { internal }) \mid=0,1 \quad(i=1,2,3) .
$$

If $\Delta_{i}=0$ then the Higgs triplets, $D_{i}$ and $\bar{D}_{i}$, remain in the massless spectrum while the Higgs doublets, $h_{i}$ and $\bar{h}_{i}$ are projected out and the opposite occurs for $\Delta_{i}=1$.

The rule in Eq. (2.5) is a generic rule that operates in NAHE-based free fermionic models. The model of table 2.2 illustrates this rule. In this model the basis vector that breaks $S O(10)$ symmetry to $S O(6) \times S O(4)$ is $\alpha$ and, with respect to $\alpha$, $\Delta_{1}=\Delta_{2}=\Delta_{3}=1$. Therefore, this model produces three pairs of electroweak Higgs doublets from the Neveu-Schwarz sector, $h_{1}, \bar{h}_{1} h_{2}, \bar{h}_{2}$ and $h_{3}, \bar{h}_{3}$, and all the untwisted colour triplets are projected out. Note also that the vector basis $b_{4}$ is symmetric with respect to the internal fermions that are periodic in the vectors $b_{i}, i=1,2,3$ and, therefore, does not project out the fields in the 10 representation of $S O(10)$.

Another relevant question with regard to the Higgs doublet-triplet splitting mechanism is whether it is possible to construct models in which both the Higgs colour triplets and electroweak doublets from the Neveu-Schwarz sector are projected out by the GSO projections. This is a viable possibility as we can choose for example

$$
\Delta_{j}^{(\alpha)}=1 \text { and } \Delta_{j}^{(\beta)}=0,
$$

where $\Delta^{(\alpha, \beta)}$ are the projections due to the basis vectors $\alpha$ and $\beta$ respectively. This is a relevant question as the number of Higgs representations, which generically appear in the massless spectrum, is larger than what is allowed by the low energy phenomenology. Attempts to construct such models were discussed in ref. [19]. However, in the models presented there this was achieved at the expense of projecting some of the states from the sectors $b_{1}, b_{2}$ and $b_{3}$. In section 3 we present for the first time three generation models with reduced untwisted Higgs spectrum, without resorting to analysis of supersymmetric flat directions. 


\section{Models with reduced untwisted Higgs spectrum}

As an illustration of the Higgs reduction mechanism we consider the model in table 3.1

\begin{tabular}{|c|c|c|c|c|c|c|c|c|c|}
\hline & $\psi^{\mu}$ & $\chi^{12}$ & $\chi^{34}$ & $\chi^{56}$ & $\bar{\psi}^{1, \ldots, 5}$ & $\bar{\eta}^{1}$ & $\bar{\eta}^{2}$ & $\bar{\eta}^{3}$ & $\bar{\phi}^{1, \ldots, 8}$ \\
\hline$\alpha$ & $\overline{c 1}$ & $\overline{c 1}$ & 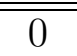 & 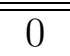 & $\begin{array}{lllll}1 & 1 & 1 & 0 & 0\end{array}$ & 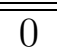 & $\overline{c 1}$ & 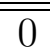 & $\begin{array}{lllllllll}0 & 1 & 1 & 0 & 0 & 0 & 0 & 0\end{array}$ \\
\hline$\beta$ & 1 & 0 & 1 & 0 & 11100 & 1 & 1 & 1 & 0111000000 \\
\hline$\gamma$ & 1 & 0 & 0 & 1 & $\frac{1}{2} \quad \frac{1}{2} \quad \frac{1}{2} \quad \frac{1}{2} \quad \frac{1}{2}$ & $\frac{1}{2}$ & $\frac{1}{2}$ & $\frac{1}{2}$ & $\frac{1}{2} 000 \frac{1}{2} \frac{1}{2} \frac{1}{2} 0$ \\
\hline
\end{tabular}

\begin{tabular}{c|cccc|cccc|cccc} 
& $y^{3} y^{6}$ & $y^{4} \bar{y}^{4}$ & $y^{5} \bar{y}^{5} \bar{y}^{3} \bar{y}^{6}$ & \multicolumn{1}{|c|}{$y^{1} \omega^{6} y^{2} \bar{y}^{2} \omega^{5} \bar{\omega}^{5} \bar{y}^{1} \bar{\omega}^{6}$} & $\omega^{1} \omega^{3} \omega^{2} \bar{\omega}^{2} \omega^{4} \bar{\omega}^{4} \bar{\omega}^{1} \bar{\omega}^{3}$ \\
\hline \hline$\alpha$ & 1 & 0 & 0 & 0 & 0 & 0 & 1 & 1 & 0 & 0 & 1 & 0 \\
$\beta$ & 0 & 0 & 1 & 1 & 1 & 0 & 0 & 1 & 0 & 1 & 0 & 1 \\
$\gamma$ & 0 & 1 & 0 & 1 & 0 & 1 & 0 & 0 & 1 & 0 & 0 & 1
\end{tabular}

With the choice of generalised GSO coefficients:

$$
\begin{aligned}
& c\left(\begin{array}{c}
\alpha, \beta \\
\alpha
\end{array}\right)=c\left(\begin{array}{c}
\beta, \gamma \\
\beta
\end{array}\right)=-c\left(\begin{array}{c}
\gamma \\
\mathbf{1}, \alpha
\end{array}\right)=c\left(\begin{array}{c}
\alpha \\
b_{3}
\end{array}\right)= \\
& c\left(\begin{array}{c}
\gamma \\
b_{1}
\end{array}\right)=-c\left(\begin{array}{c}
\beta \\
b_{j}
\end{array}\right)=-c\left(\begin{array}{c}
\alpha \\
b_{1}, b_{2}
\end{array}\right)=-c\left(\begin{array}{c}
\gamma \\
b_{2}, b_{3}
\end{array}\right)=1
\end{aligned}
$$

$(j=1,2,3)$, with the others specified by modular invariance and space-time supersymmetry. As noted from the table, in this model the boundary conditions with respect to $b_{2}$ and $b_{3}$ in the basis vector $\alpha$ are asymmetric and symmetric, respectively, while the opposite occurs for the basis vector $\beta$. At the same time, the boundary conditions with respect to the sector $b_{1}$ are asymmetric in both $\alpha$ and $\beta$. Therefore, in this model $\Delta_{1}^{(\alpha)}=\Delta_{1}^{(\beta)}=1 ; \Delta_{2}^{(\alpha)}=1, \Delta_{2}^{(\beta)}=0$ and $\Delta_{3}^{(\alpha)}=0, \Delta_{3}^{(\beta)}=1$. Consequently, in this model, irrespective of the choice of the generalised GSO projection coefficients, both the Higgs colour triplets and electroweak doublets associated with $b_{2}$ and $b_{3}$ are projected out by the GSO projections, whereas the electroweak Higgs doublets that are associated with the sector $b_{1}$ remain in the spectrum. However, in this model, the sector $\alpha$ produces chiral fractionally charged exotics, and is therefore not viable. We also note that in this model the non-vanishing cubic level Yukawa couplings produce a down-quark type mass term, and not a potential top-quark mass term.

\begin{tabular}{|c|c|c|c|c|c|c|c|c|c|}
\hline & $\psi^{\mu}$ & $\chi^{12}$ & $\chi^{34}$ & $\chi^{56}$ & $\bar{\psi}^{1, \ldots, 5}$ & $\bar{\eta}^{1}$ & $\bar{\eta}^{2}$ & $\bar{\eta}^{3}$ & $\bar{\phi}^{1, \ldots, 8}$ \\
\hline$b_{4}$ & 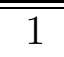 & $\overline{c 1}$ & "0 & 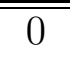 & $\begin{array}{lllll} & 1 & 1 & 1 & 1\end{array}$ & 0 & $\overline{c 1}$ & 0 & 11111100000 \\
\hline$\beta$ & 1 & 0 & 1 & 0 & 11100 & 1 & 1 & 1 & $\begin{array}{llllllll}0 & 0 & 0 & 0 & 1 & 1 & 0 & 0\end{array}$ \\
\hline$\gamma$ & 1 & 0 & 0 & 1 & $\begin{array}{lllll}\frac{1}{2} & \frac{1}{2} & \frac{1}{2} & \frac{1}{2} & \frac{1}{2}\end{array}$ & $\frac{1}{2}$ & $\frac{1}{2}$ & $\frac{1}{2}$ & $00 \frac{1}{2} \frac{1}{2} 00 \frac{1}{2} \frac{1}{2}$ \\
\hline
\end{tabular}

An alternative model is presented in table 3.2 


\begin{tabular}{c|cccc|cccc|rccc} 
& $y^{3} y^{6} y^{4} \bar{y}^{4}$ & $y^{5} \bar{y}^{5}$ & $\bar{y}^{3} \bar{y}^{6}$ & \multicolumn{1}{|c|}{$y^{1} \omega^{6}$} & $y^{2} \bar{y}^{2}$ & $\omega^{5} \bar{\omega}^{5}$ & $\bar{y}^{1} \bar{\omega}^{6}$ & $\omega^{1} \omega^{3} \omega^{2} \bar{\omega}^{2} \omega^{4} \bar{\omega}^{4} \bar{\omega}^{1} \bar{\omega}^{3}$ \\
\hline \hline$b_{4}$ & 1 & 0 & 0 & 0 & 0 & 0 & 1 & 1 & 0 & 0 & 1 & 0 \\
$\beta$ & 0 & 0 & 1 & 1 & 1 & 0 & 0 & 1 & 0 & 1 & 0 & 1 \\
$\gamma$ & 0 & 1 & 0 & 1 & 0 & 0 & 0 & 1 & 1 & 1 & 0 & 0
\end{tabular}

With the choice of generalised GSO coefficients:

$$
\begin{aligned}
& c\left(\begin{array}{c}
b_{4} \\
b_{4}, \beta, \gamma
\end{array}\right)=c\left(\begin{array}{c}
\beta \\
\beta, \gamma
\end{array}\right)=c\left(\begin{array}{c}
b_{4}, \gamma \\
b_{j}
\end{array}\right)= \\
& -c\left(\begin{array}{l}
\gamma \\
\mathbf{1}
\end{array}\right)=-c\left(\begin{array}{l}
\beta \\
b_{j}
\end{array}\right)=1
\end{aligned}
$$

$(\mathrm{j}=1,2,3)$, with the others specified by modular invariance and space-time supersymmetry. In this model the basis vector $b_{4}$ preserves the $S O(10)$ symmetry, which is broken by the basis vectors $\beta$ and $\gamma$ to $S U(3) \times S U(2) \times U(1)^{2}$. The $b_{4}$ projection is asymmetric with respect to the internal fermions that are periodic in the sectors $b_{1}$ and $b_{2}$ and, therefore, projects out the entire untwisted vectorial representations of $S O(10)$, that couple to the sectors $b_{1}$ and $b_{2}$, irrespective of the $\beta$ projection. On the other hand, it is symmetric with respect to $b_{3}$, while the basis vector $\beta$, that breaks $S O(10) \rightarrow S O(6) \times S O(4)$, is asymmetric with respect to $b_{3}$. Therefore, the Higgs doublets that couple to $b_{3}$ remain in the massless spectrum. We note also that the boundary conditions in the vector $\gamma$, that breaks $S O(10) \rightarrow S U(5) \times U(1)$, are asymmetric with respect to the internal fermions that are periodic in the sector $b_{3}$. Therefore, this model will select an up-quark type Yukawa couplings at the cubic level of the superpotential. The gauge group of this model is generated entirely from the untwisted vector bosons and there is no gauge symmetry enhancement from additional sectors. The four dimensional gauge group is $S U(3)_{C} \times S U(2)_{L} \times U(1)_{B-L} \times U(1)_{T_{3}} \times U(1)_{1, \cdots, 6} \times S U(2)_{1, \cdots, 6} \times U(1)_{7,8}$.

The spectrum of the model is detailed in the table 4.1 at the end of this paper. The cubic level superpotential, including states from the observable and hidden sectors, is straightforwardly calculated following the rules given in [14] and reads:

$$
\begin{aligned}
W & =N_{L_{3}}^{c} L_{3} \bar{h}+u_{L_{3}}^{c} Q_{3} \bar{h}+C_{+}^{-+} D_{-} \bar{h}+C_{-}^{+-} D_{+} h+ \\
& +\left(\phi_{1} \phi_{3}{ }^{\prime}+\phi_{1}^{\prime} \phi_{3}\right) \phi_{2}+\left(C_{+}^{-+} C_{-}^{+-}+C_{-}^{-+} C_{+}^{+-}\right) \phi_{3}^{\prime} \\
& +\left(D_{+} D_{-}+C_{+} C_{-}+T_{+} T_{-}+D_{+-}^{(6)} D_{-+}^{(6)}+D_{--}^{(6)} D_{++}^{(6)}\right) \phi_{3} \\
& +\left(D_{+-}^{(3,4)} D_{-+}^{(3,4)}+D_{+}^{(5)} D_{-}^{(5)}+D_{++}^{(3)} D_{--}^{(3)}+D_{+-}^{(3)} D_{-+}^{(3)}\right) \phi_{1} \\
& +A_{+} A_{-} \phi_{1}^{\prime} .
\end{aligned}
$$

As expected, we obtain a Yukawa coupling for the top quark, but also couplings of the Higgs with exotic states. One can also see that not all the fractionally charged ${ }^{1}$ states in the spectrum appear in the cubic level superpotential, which means that they remain massless at the trilinear level. However, this does not exclude the possibility of giving them masses at higher orders.

\footnotetext{
${ }^{1}$ The hypercharge is defined as $Q_{Y}=1 / 3 Q_{C}+1 / 2 Q_{L}$ and the electric charge is given by $Q_{e}=T_{3 L}+Q_{Y}$, with $T_{3 L}$ the electroweak isospin.
} 


\subsection{Flat directions}

In this section we investigate the flat directions of the model of table 3.2. The model contains 6 anomalous $U(1)$ 's with

$$
\begin{aligned}
& \operatorname{Tr} Q_{1}=\operatorname{Tr} Q_{2}=-\operatorname{Tr} Q_{3}=\operatorname{Tr} Q_{5}=-24 \\
& \operatorname{Tr} Q_{4}=-\operatorname{Tr} Q_{6}=12 .
\end{aligned}
$$

The total anomaly can be rotated into a single $U(1)_{A}$ and the new basis reads

$$
\begin{aligned}
Q_{1}^{\prime} & =Q_{1}-Q_{2}, \\
Q_{2}^{\prime} & =Q_{3}+Q_{5} \\
Q_{3}^{\prime} & =Q_{4}+Q_{6} \\
Q_{4}^{\prime} & =Q_{1}+Q_{2}+Q_{3}-Q_{5} \\
Q_{5}^{\prime} & =Q_{1}+Q_{2}-Q_{3}+Q_{5}+4\left(Q_{4}-Q_{6}\right) \\
Q_{A} & =2\left(Q_{1}+Q_{2}-Q_{3}+Q_{5}\right)-Q_{4}+Q_{6}
\end{aligned}
$$

In the following we will call $Q_{i}^{\prime}, \mathrm{i}=1, \ldots, 5$, simply $Q_{i}$.

To search for flat directions we use the methodology developed in [20]. We start by constructing a basis of D-flat directions under $Q_{1 \ldots 5}$ and then we investigate the existence of D-flat directions in the anomalous $U(1)_{A}$. Subsequently we will have to impose D-flatness under the remaining gauge groups and F-flatness. To generate the basis of flat directions under $Q_{1 \ldots 5}$ we start by forming a basis of gauge invariant monomials under $U(1)_{1}$, then we use these invariants to construct a basis of invariant monomials under $U(1)_{2}$ and so forth.

We include in the analysis only the fields with vanishing hypercharge ${ }^{1}$ and which are singlets under the Standard Model gauge group. The $Q_{1 \ldots 5, A}$ charges of these fields are detailed in table 3.6, where, following the notation of [20], we signal by ${ }^{(\prime)}\left({ }^{\left({ }^{\prime \prime}\right)}\right)$ the presence in the spectrum of a second (third) field with the same $U(1)_{1 \ldots 5, A}$ charges and by $\sqrt{ }$ the presence of a field with opposite $U(1)_{1 \ldots 5, A}$ charges. For instance, the field $\phi$ stands for $\phi_{1}$, while $\phi^{\prime}$ stands for $\phi_{3}$ and the two fields with opposite charges are $\phi_{1}^{\prime}$ and $\phi_{3}^{\prime}$. The fields with opposite charges to $A_{+}$and $A_{-}$are $D_{-}^{(5)}$ and $D_{+}^{(5)}$, respectively, while the field with opposite charges to $D_{2}$ is $D_{+-}^{(3,4)}$ and $\tilde{D}_{2}^{\prime \prime}$ stands for $D_{-+}^{(3,4)}$, in the notation of the table 4.1. We did not include in table 3.6 the fields $\tilde{\phi}_{1}$, $\phi_{2}$ and $\tilde{\phi}_{3}$, which have vanishing charges. These fields are trivially flat directions in the $U(1)_{1 \ldots 5}$, but they are not flat under the anomalous $U(1)$.

For simplicity we rescaled the charges $Q_{1}, Q_{3}$ and $Q_{A}$ by a factor 2 and the charges $Q_{2}, Q_{4}$ and $Q_{5}$ by a factor 4 . The seventh column is given by

$$
\hat{Q}=\frac{1}{18}\left(Q_{A}-Q_{5}+9 Q_{3}\right)
$$

and, as explained in [20], it will be useful for the search of flat directions in the anomalous $U(1)$. 


\begin{tabular}{l|rrrrrrr} 
& $Q_{1}$ & $Q_{2}$ & $Q_{3}$ & $Q_{4}$ & $Q_{5}$ & $Q_{A}$ & $\hat{Q}$ \\
\hline \hline$\phi^{\left({ }^{\prime}\right)} \sqrt{ }^{\left({ }^{\prime}\right)}$ & 0 & 4 & 0 & -4 & 4 & 4 & 0 \\
$S_{1}^{\left({ }^{\prime}\right)}, D_{1}$ & 1 & 2 & -1 & 0 & -12 & -3 & 0 \\
$\tilde{S}_{1}^{\left({ }^{\prime}\right)}, \tilde{D}_{1}^{\left({ }^{\prime}\right)}$ & 1 & 2 & 1 & 0 & 4 & -5 & 0 \\
$S_{2}^{\left({ }^{\prime}\right)}, D_{2} \sqrt{ }$ & -1 & 4 & 0 & -2 & -2 & -2 & 0 \\
$\tilde{S}_{2}^{\left({ }^{\prime}\right)}, \tilde{D}_{2}^{\left({ }^{\prime}\right)\left({ }^{\prime \prime}\right)}$ & -1 & 0 & 0 & 2 & -6 & -6 & 0 \\
$S_{3}^{\left({ }^{\prime}\right)}, D_{3}$ & 0 & 0 & 1 & -4 & -12 & -3 & 1 \\
$\tilde{S}_{3}^{\left({ }^{\prime}\right)}, \tilde{D}_{3}^{\left({ }^{\prime}\right)}$ & 0 & 0 & -1 & -4 & 4 & -5 & -1 \\
$N_{1}$ & -1 & 0 & -1 & -2 & -10 & -1 & 0 \\
$N_{2}$ & 1 & -2 & 0 & 0 & -4 & -4 & 0 \\
$N_{3}$ & 0 & 2 & -1 & 2 & 6 & -3 & -1 \\
$A_{+} \sqrt{ }$ & -1 & 0 & 0 & -6 & 2 & 2 & 0 \\
$A_{-} \sqrt{ }$ & 1 & 4 & 0 & 2 & 2 & 2 & 0 \\
$F^{\left({ }^{\prime}\right)}$ & 1 & -1 & 2 & -1 & 1 & 1 & 1 \\
$\tilde{F}^{\left({ }^{\prime}\right)}$ & -1 & 1 & 0 & 1 & 15 & -3 & -1 \\
$F_{1}$ & 0 & 3 & 1 & 1 & 11 & 2 & 0 \\
$F_{2}$ & 0 & -1 & 1 & 5 & 7 & -2 & 0 \\
$F_{3}$ & 0 & 1 & 1 & -5 & 9 & 0 & 0 \\
$F_{4}$ & 0 & -3 & 1 & -1 & 5 & -4 & 0
\end{tabular}

As a first step we investigate the existence of flat directions involving vacuum expectation values only for the fields which are singlets under both the visible and the hidden gauge groups. These fields are $\phi^{\left({ }^{\prime}\right)} \mathfrak{V}^{\left({ }^{\prime}\right)}, S_{1}^{\left({ }^{\prime}\right)}, \tilde{S}_{1}^{\left({ }^{\prime}\right)}, S_{2}^{\left({ }^{\prime}\right)}, \tilde{S}_{2}^{\left({ }^{\prime}\right)}, S_{3}^{\left({ }^{\prime}\right)}, \tilde{S}_{3}^{\left({ }^{\prime}\right)}, N_{1}$, $N_{2}$ and $N_{3}$. Bearing in mind the equivalence in the charges for some fields, these count as 11 fields and so, given the fact that we have to impose 5 constraints, the basis of flat directions should contain 6 elements. But a simple Mathematica program can show that it is impossible to incorporate the fields $S_{1}^{\left({ }^{\prime}\right)}, S_{3}^{\left({ }^{\prime}\right)}, N_{1}, N_{2}$ and $N_{3}$ into the flat directions. This leave us with 6 fields, so we expect a basis with just one element. It turns out that, in respect with the charges of the remaining fields, $Q_{4}$ and $Q_{5}$ are a linear combination of the previous $U(1)$ 's, so there are actually only 3 independent constraints and, hence, we obtain three basis elements

$$
\phi \bar{\phi}, \quad \bar{\phi} \tilde{S}_{1}^{2} \tilde{S}_{2}^{2} \tilde{S}_{3}^{2}, \quad \bar{\phi}^{3} \tilde{S}_{1}^{2} S_{2}^{2} \tilde{S}_{3}^{2}
$$

where we expressed the flat directions as gauge invariant monomials. For example, the monomial $\bar{\phi} \tilde{S}_{1}^{2} \tilde{S}_{2}^{2} \tilde{S}_{3}^{2}$ corresponds to the following choice of VEVs

$$
|\bar{\phi}|^{2}=|\psi|^{2},\left|\tilde{S}_{1}\right|^{2}=2|\psi|^{2},\left|\tilde{S}_{2}\right|^{2}=2|\psi|^{2},\left|\tilde{S}_{3}\right|^{2}=2|\psi|^{2}
$$

for an arbitrary $|\psi|$.

Note that in the precedent basis any field A can be replaced with its copy A'. Any flat direction, $P$, can be obtained from the elements of the basis as 


$$
P^{n}=\prod_{\alpha} M_{\alpha}^{n_{\alpha}}
$$

where $M_{\alpha}$ stand for the elements of the basis, $n$ is a positive integer and $n_{\alpha}$ are integers [20].

In order to obtain D-flat directions in the anomalous $U(1)$ we need to construct invariant monomials containing the field $S_{3}^{\left({ }^{\prime}\right)}$, since this is the only field with a positive $\hat{Q}$ charge ${ }^{1}$, necessary to cancel the negative Fayet-Iliopoulos term generated by the anomalous $U(1)^{2}$. And, since none of the elements of the basis contains this field, we conclude that there are no flat directions involving only VEVs of the singlets.

Therefore, we proceed with the analysis including also nonabelian fields under the hidden gauge group. This amounts to including all the fields in table 3.6. which contains 22 fields with non-equivalent charges. Again, we look for a basis of gauge invariant monomials under $Q_{1 \ldots 5}$. Such a basis is given by

$$
\begin{aligned}
& \phi \bar{\phi}, \quad D_{2} \bar{D}_{2}, \quad A_{+} \bar{A}_{+}, \quad A_{-} \bar{A}_{-}, \quad \bar{\phi} \tilde{S}_{1}^{2} \tilde{S}_{2}^{2} \tilde{S}_{3}^{2}, \quad \bar{\phi}^{3} \tilde{S}_{1}^{2} S_{2}^{2} \tilde{S}_{3}^{2}, \quad \bar{\phi} A_{+} A_{-}, \\
& \bar{\phi} S_{1}^{4} N_{1}^{2} F^{2} \tilde{F}^{4} F_{4}^{2}, \quad \bar{\phi} S_{1}^{2} S_{3}^{2} N_{3}^{2} \tilde{F}^{2} F_{4}^{2}, \quad \bar{\phi} S_{3}^{2} N_{1}^{2} N_{2}^{2} N_{3}^{4} F^{2} \tilde{F}^{2}, \\
& \bar{\phi} S_{3}^{2} N_{1}^{2} N_{2}^{2} N_{3}^{4} F_{1}^{2} F_{4}^{2}, \quad \bar{\phi} S_{3}^{2} N_{1}^{2} N_{2}^{2} N_{3}^{4} F_{2}^{2} F_{3}^{2}, \quad S_{1}^{2} \tilde{S}_{2} S_{3} \tilde{S}_{3} \bar{A}_{+} \tilde{F}^{2} F_{4}^{2}, \\
& S_{1}^{3} \tilde{S}_{2}^{3} S_{3} \tilde{S}_{3}^{2} N_{1} N_{2} \bar{A}_{+}^{3} \tilde{F}^{3} F_{3}^{2} F_{4}^{3}, \quad \tilde{S}_{2}^{5} S_{3} \tilde{S}_{3}^{5} \bar{A}_{+}^{5} F_{3}^{3} F_{4}, \quad \bar{\phi} S_{1}^{10} \tilde{S}_{2}^{2} S_{3}^{2} \tilde{F}^{8} F_{4}^{8}, \\
& S_{1}^{9} \tilde{S}_{2}^{2} S_{3}^{2} N_{1} N_{2} \bar{A}_{+} \tilde{F}^{8} F_{4}^{8}
\end{aligned}
$$

where, again, any field can be replaced with one of its copies with equal $Q_{1 \ldots 5}$ charges. All the elements of the basis have negative or vanishing $\hat{Q}$ charges, but, since some of the elements contain the fields $S_{3}$ and $F$, which have positive $\hat{Q}$ charge, and, since flat directions can be obtained as a combination of the basis elements with negative powers, we cannot conclude immediately that there are no D-flat directions under the anomalous $U(1)$. Nevertheless, a simple Mathematica program shows that it is impossible to obtain viable invariant monomials with positive $\hat{Q}$ charge, by viable meaning that the fields that do not have a partner field with opposite charges should appear with positive powers in the monomials. We conclude that there are no flat directions involving only singlets of the visible gauge group.

Therefore, the only possibility to obtain flat directions which do not break electric charge is to consider the option of giving a VEV also to the neutral component of the

\footnotetext{
${ }^{1}$ The $\hat{Q}$ charge of an invariant monomial is equal, up to positive factors, with his $Q_{A}$ charge, since the difference between the two is a linear combination of $Q_{1 \ldots 5}$, under which the invariant monomials have zero charge by construction.

${ }^{2}$ In our model $\operatorname{Tr} Q_{A}<0$.
} 
Higgs field, in which case the flat directions would break the electroweak symmetry. The Higgs doublets in our model have the following charges:

\begin{tabular}{r|rrrrrrr} 
& $Q_{1}$ & $Q_{2}$ & $Q_{3}$ & $Q_{4}$ & $Q_{5}$ & $Q_{A}$ & $\hat{Q}$ \\
\hline \hline$h \sqrt{ }$ & 0 & 4 & 0 & 4 & -4 & -4 & 0
\end{tabular}

and including them into our analysis amounts to adding the invariant monomial $\bar{\phi} h S_{1}^{2} S_{3} \tilde{S}_{3} \tilde{F}^{2} F_{4}^{2}$ to the basis 3.10. The new basis element also has a negative $\hat{Q}$ charge and, again, it turns out to be impossible to construct flat directions with

positive $\hat{Q}$ charge. This means that the only stable vacuum solutions of our model are the ones that break the Standard Model gauge group. We did not search for such flat directions (i.e. including in the analysis all the states in the spectrum), but preliminary analysis indicates their existence.

\section{Conclusions}

The quasi-realistic free fermionic models, which are related to $Z_{2} \times Z_{2}$ orbifold compactifications at special points in the moduli space [21], produced some of the most realistic string models constructed to date. The underlying $Z_{2} \times Z_{2}$ orbifold structure dictates that the models generically contain three pairs of untwisted Higgs multiplets. The reduction of the Higgs states to the Standard Model spectrum relied in the past on the analysis of supersymmetric flat directions, which give superheavy mass to the dispensable Higgs respresentations. In this paper we investigated the possibility of removing the extra Higgs multiplets by using the free fermion boundary conditions and directly at the string level, rather than in the effective low energy field theory.

The mechanism presented relies on the possibility of assigning boundary conditions that are asymmetric between the left- and right-moving internal fermions. In this respect, it should be noted that all $Z_{2} \times Z_{2}$ orbifold models constructed to date use symmetric boundary conditions and is therefore of immense interest to incorporate asymmetric twistings in these bosonic constructions.

We also incorporated the Higgs reduction mechanism in a three generation model and therefore obtained for the first time a model with three generations of chiral fermions, arising from the 16 spinorial representation of $S O(10)$, and one pair of electroweak Higgs doublet, arising from the 10 vectorial representation of $S O(10)$, directly at the string level.

An additional effect of the asymmetric twisting is the reduction of the moduli space. In the past this has been demonstrated with respect to the untwisted moduli that parametrise the shape and size of the dimensional compactified manifold [22]. The effect of the asymmetric boundary conditions that result in untwisted Higgs reduction is, additionally, to reduce the untwisted Standard Model singlet spectrum. The consequence is that the supersymmetric moduli space is more restrictive. In fact, we demonstrated that in the model presented here there do not exist flat directions 
that preserve the Standard Model gauge group. This is in fact a welcomed situation, as it is likely that in the vast space of three generation free fermionic models [23] there do exist vacua that accommodate a viable spectrum with the Higgs reduction mechanism articulated here, and supersymmetric flat directions. In such models, however, the supersymmetric moduli space will be much reduced, hence increasing their predictive power.

\section{Acknowledgments}

AEF would like to thank the CERN and Oxford theory departments for hospitality. CT would like to thank Matteo Cardella and Thomas Mohaupt for useful discussions. This work was supported by the PPARC and the University of Liverpool.

\section{References}

[1] For reviews and references see e.g.:

P. Langacker, Phys. Rep. 72 (1981) 185;

C. Kounnas, A. Masiero, D.V. Nanopoulos and K.A. Olive, Grand Unification With And Without Supersymmetry And Cosmological Implications (World Scientific, Singapore, 1984).

[2] D.J. Gross, J.A. Harvey, J.A. Martinec and R. Rohm, Nucl. Phys. B 256 (1986) 253;

P. Candelas, G.T. Horowitz, A. Strominger and E. Witten, Nucl. Phys. B 258 (1985) 46.

[3] See e.g.: J. Giedt, Annals Phys. 297 (2002) 67;

S. Forste, H.P. Nilles, P.K.S. Vaudrevange and A. Wingerter, Phys. Rev. D 70 (2004) 106008;

T. Kobayashi, S. Raby and R.J. Zhang, Nucl. Phys. B 704 (2005) 3;

V. Bouchard and R. Donagi, Phys. Lett. B 633 (2006) 783;

V. Braun, Y.H. He, B.A. Ovrut and T. Pantev, JHEP 0605, (2006) 043;

R. Blumenhagen, S. Moster and T. Weigand, Nucl. Phys. B 751 (2006) 186;

W. Buchmuller, K. Hamaguchi, O. Lebedev and M. Ratz, Phys. Rev. Lett. 96 (2006) 121602;

J. E. Kim and B. Kyae, arXiv:hep-th/0608085,

J. E. Kim and B. Kyae, arXiv:hep-th/0608086.

[4] I. Antoniadis, J. Ellis, J. Hagelin and D.V. Nanopoulos Phys. Lett. B 231 (1989) 65 ;

J.L. Lopez, D.V. Nanopoulos and K. Yuan, Nucl. Phys. B 399 (1993) 3.

[5] A.E. Faraggi, D.V. Nanopoulos and K. Yuan, Nucl. Phys. B 335 (1990) 347. 
[6] A.E. Faraggi, Phys. Rev. D 46 (1992) 3204.

[7] I. Antoniadis, G.K. Leontaris and J. Rizos, Phys. Lett. B 245 (1990) 161;

G.K. Leontaris and J. Rizos, Nucl. Phys. B 554 (1999) 3;

[8] A.E. Faraggi, Phys. Lett. B 278 (1992) 131; Nucl. Phys. B 387 (1992) 239; Nucl. Phys. B 403 (1993) 101.

[9] A.E. Faraggi, Phys. Lett. B 274 (1992) 47; Phys. Rev. D 47 (1993) 5021; Phys. Lett. B 377 (1996) 43; Nucl. Phys. B 487 (1997) 55.

[10] A.E. Faraggi and D.V. Nanopoulos, Phys. Rev. D 48 (1993) 3288;

A.E. Faraggi, Nucl. Phys. B 387 (1992) 239.

[11] G.B. Cleaver, A.E. Faraggi and D.V. Nanopoulos, Phys. Lett. B 455 (1999) 135; Int. J. Mod. Phys. A 16 (2001) 425;

G.B. Cleaver, A.E. Faraggi, D.V. Nanopoulos and J.W. Walker, Nucl. Phys. B 593 (2001) 471; Nucl. Phys. B 620 (2002) 259.

[12] G.B. Cleaver, A.E. Faraggi and C. Savage, Phys. Rev. D 63 (2001) 066001;

G.B. Cleaver, D.J. Clements and A.E. Faraggi, Phys. Rev. D 65 (2002) 106003.

[13] H. Kawai, D.C. Lewellen, and S.H.-H. Tye, Nucl. Phys. B 288 (1987) 1;

I. Antoniadis, C. Bachas, and C. Kounnas, Nucl. Phys. B 289 (1987) 87;

I. Antoniadis and C. Bachas, Nucl. Phys. B 289 (1987) 87.

[14] S. Kalara, J.L. Lopez and D.V. Nanopoulos, Nucl. Phys. B 353 (1991) 650.

[15] S. Ferrara, L. Girardello, C. Kounnas and M. Porrati, Phys. Lett. B 194 (1987) 368 ;

S. Ferrara, C. Kounnas, M. Porrati and F. Zwirner, Phys. Lett. B 194 (1987) 366.

[16] G.B. Cleaver, A.E. Faraggi and S.E.M. Nooij, Nucl. Phys. B 672 (2003) 64.

[17] M. Dine, N. Seiberg and E. Witten, Nucl. Phys. B 289 (1987) 585.

[18] A.E. Faraggi, Nucl. Phys. B 428 (1994) 111; Phys. Lett. B 520 (2001) 337.

[19] A.E. Faraggi, Int. J. Mod. Phys. A 14 (1999) 1663.

[20] G. Cleaver, M. Cvetic, J. R. Espinosa, L. L. Everett, P. Langacker and J. Wang, Phys. Rev. D 59 (1999) 115003 
[21] A.E. Faraggi, Phys. Lett. B 326 (1994) 62; hep-th/9511093;

J. Ellis, A.E. Faraggi and D.V. Nanopoulos, Phys. Lett. B 419 (1998) 123;

P. Berglund, J. Ellis, A.E. Faraggi, D.V. Nanopoulos and Z. Qiu, Phys. Lett. B 433 (1998) 269; Int. J. Mod. Phys. A 15 (2000) 1345;

A.E. Faraggi, Phys. Lett. B 544 (2002) 207; hep-th/0411118;

A.E. Faraggi and R. Donagi, Nucl. Phys. B 694 (2004) 187;

A.E. Faraggi, S. Förste, M.C. Timirgaziu, JHEP 0608, (2006) 057.

[22] A.E. Faraggi, Nucl. Phys. B 728 (2005) 83.

[23] A.E. Faraggi, C. Kounnas, S. Nooij and J. Rizos, hep-th/0311058; Nucl. Phys. B 695 (2004) 41; hep-th/0606144. 
Table 4.1

\begin{tabular}{|c|c|c|c|c|c|c|c|c|c|c|c|c|c|}
\hline$F$ & SEC & $\begin{array}{c}S U(3) \times \\
S U(2) \\
\end{array}$ & $Q_{C}$ & $Q_{L}$ & $Q_{1}$ & $Q_{2}$ & $Q_{3}$ & $Q_{4}$ & $Q_{5}$ & $Q_{6}$ & $S U(2)_{1, . .6}^{6}$ & $Q_{7}$ & $Q_{8}$ \\
\hline$L_{1}$ & $b_{1}$ & $(1,2)$ & $-\frac{3}{2}$ & 0 & $-\frac{1}{2}$ & 0 & 0 & $-\frac{1}{2}$ & 0 & 0 & $(1,1,1,1,1,1)$ & 0 & 0 \\
\hline$Q_{1}$ & & $(3,2)$ & $\frac{1}{2}$ & 0 & $-\frac{1}{2}$ & 0 & 0 & $\frac{1}{2}$ & 0 & 0 & $(1,1,1,1,1,1)$ & 0 & 0 \\
\hline$d_{L_{1}}^{c}$ & & $(\overline{3}, 1)$ & $-\frac{1}{2}$ & 1 & $-\frac{1}{2}$ & 0 & 0 & $-\frac{1}{2}$ & 0 & 0 & $(1,1,1,1,1,1)$ & 0 & 0 \\
\hline$N_{L_{1}}^{c}$ & & $(1,1)$ & $\frac{3}{2}$ & -1 & $-\frac{1}{2}$ & 0 & 0 & $-\frac{1}{2}$ & 0 & 0 & $(1,1,1,1,1,1)$ & 0 & 0 \\
\hline$u_{L_{1}}^{c}$ & & $(\overline{3}, 1)$ & $-\frac{1}{2}$ & -1 & $-\frac{1}{2}$ & 0 & 0 & $\frac{1}{2}$ & 0 & 0 & $(1,1,1,1,1,1)$ & 0 & 0 \\
\hline$e_{L_{1}}^{c}$ & & $(1,1)$ & $\frac{3}{2}$ & 1 & $-\frac{1}{2}$ & 0 & 0 & $\frac{1}{2}$ & 0 & 0 & $(1,1,1,1,1,1)$ & 0 & 0 \\
\hline$L_{2}$ & $b_{2}$ & $(1,2)$ & $-\frac{3}{2}$ & 0 & 0 & $-\frac{1}{2}$ & 0 & 0 & $\frac{1}{2}$ & 0 & $(1,1,1,1,1,1)$ & 0 & 0 \\
\hline$Q_{2}$ & & $(3,2)$ & $\frac{1}{2}$ & 0 & 0 & $-\frac{1}{2}$ & 0 & 0 & $-\frac{1}{2}$ & 0 & $(1,1,1,1,1,1)$ & 0 & 0 \\
\hline$d_{L_{2}}^{c}$ & & $(\overline{3}, 1)$ & $-\frac{1}{2}$ & 1 & 0 & $-\frac{1}{2}$ & 0 & 0 & $-\frac{1}{2}$ & 0 & $(1,1,1,1,1,1)$ & 0 & 0 \\
\hline$N_{L_{2}}^{c}$ & & $(1,1)$ & $\frac{3}{2}$ & -1 & 0 & $-\frac{1}{2}$ & 0 & 0 & $-\frac{1}{2}$ & 0 & $(1,1,1,1,1,1)$ & 0 & 0 \\
\hline$u_{L_{2}}^{c}$ & & $(\overline{3}, 1)$ & $-\frac{1}{2}$ & -1 & 0 & $-\frac{1}{2}$ & 0 & 0 & $\frac{1}{2}$ & 0 & $(1,1,1,1,1,1)$ & 0 & 0 \\
\hline$e_{L_{2}}^{c}$ & & $(1,1)$ & $\frac{3}{2}$ & 1 & 0 & $-\frac{1}{2}$ & 0 & 0 & $\frac{1}{2}$ & 0 & $(1,1,1,1,1,1)$ & 0 & 0 \\
\hline$L_{3}$ & $b_{3}$ & $(1,2)$ & $-\frac{3}{2}$ & 0 & 0 & 0 & $\frac{1}{2}$ & 0 & 0 & $\frac{1}{2}$ & $(1,1,1,1,1,1)$ & 0 & 0 \\
\hline$Q_{3}$ & & $(3,2)$ & $\frac{1}{2}$ & 0 & 0 & 0 & $\frac{1}{2}$ & 0 & 0 & $-\frac{1}{2}$ & $(1,1,1,1,1,1)$ & 0 & 0 \\
\hline$d_{L_{3}}^{c}$ & & $(\overline{3}, 1)$ & $-\frac{1}{2}$ & 1 & 0 & 0 & $\frac{1}{2}$ & 0 & 0 & $-\frac{1}{2}$ & $(1,1,1,1,1,1)$ & 0 & 0 \\
\hline$N_{L_{3}}^{c}$ & & $(1,1)$ & $\frac{3}{2}$ & -1 & 0 & 0 & $\frac{1}{2}$ & 0 & 0 & $-\frac{1}{2}$ & $(1,1,1,1,1,1)$ & 0 & 0 \\
\hline$u_{L_{3}}^{c}$ & & $(\overline{3}, 1)$ & $-\frac{1}{2}$ & -1 & 0 & 0 & $\frac{1}{2}$ & 0 & 0 & $\frac{1}{2}$ & $(1,1,1,1,1,1)$ & 0 & 0 \\
\hline$e_{L_{3}}^{c}$ & & $(1,1)$ & $\frac{3}{2}$ & 1 & 0 & 0 & $\frac{1}{2}$ & 0 & 0 & $\frac{1}{2}$ & $(1,1,1,1,1,1)$ & 0 & 0 \\
\hline$h$ & NS & $(1,2)$ & 0 & -1 & 0 & 0 & 1 & 0 & 0 & 0 & $(1,1,1,1,1,1)$ & 0 & 0 \\
\hline $\bar{h}$ & & $(1,2)$ & 0 & 1 & 0 & 0 & -1 & 0 & 0 & 0 & $(1,1,1,1,1,1)$ & 0 & 0 \\
\hline$\phi_{1}$ & & $(1,1)$ & 0 & 0 & 0 & 0 & 0 & 0 & 1 & 0 & $(1,1,1,1,1,1)$ & 0 & 0 \\
\hline$\phi_{1}^{\prime}$ & & $(1,1)$ & 0 & 0 & 0 & 0 & 0 & 0 & -1 & 0 & $(1,1,1,1,1,1)$ & 0 & 0 \\
\hline$\tilde{\phi}_{1}$ & & $(1,1)$ & 0 & 0 & 0 & 0 & 0 & 0 & 0 & 0 & $(1,1,1,1,1,1)$ & 0 & 0 \\
\hline$\phi_{2}$ & & $(1,1)$ & 0 & 0 & 0 & 0 & 0 & 0 & 0 & 0 & $(1,1,1,1,1,1)$ & 0 & 0 \\
\hline$\phi_{3}$ & & $(1,1)$ & 0 & 0 & 0 & 0 & 0 & 0 & 1 & 0 & $(1,1,1,1,1,1)$ & 0 & 0 \\
\hline$\phi_{3}^{\prime}$ & & $(1,1)$ & 0 & 0 & 0 & 0 & 0 & 0 & -1 & 0 & $(1,1,1,1,1,1)$ & 0 & 0 \\
\hline$\tilde{\phi}_{3}$ & & $(1,1)$ & 0 & 0 & 0 & 0 & 0 & 0 & 0 & 0 & $(1,1,1,1,1,1)$ & 0 & 0 \\
\hline
\end{tabular}




\begin{tabular}{|c|c|c|c|c|c|c|c|c|c|c|c|c|c|}
\hline$F$ & SEC & $\begin{array}{c}S U(3) \times \\
S U(2)\end{array}$ & $Q_{C}$ & $Q_{L}$ & $Q_{1}$ & $Q_{2}$ & $Q_{3}$ & $Q_{4}$ & $Q_{5}$ & $Q_{6}$ & $S U(2)_{1, . ., 6}^{6}$ & $Q_{7}$ & $Q_{8}$ \\
\hline$C_{+}^{-+}$ & $1+b_{4}$ & $(1,1)$ & 0 & -1 & $-\frac{1}{2}$ & 0 & $\frac{1}{2}$ & 0 & $\frac{1}{2}$ & 0 & $(1,1,1,1,1,1)$ & 1 & 0 \\
\hline$C_{-}^{-+}$ & $+\beta+2 \gamma$ & , 1) & 0 & 1 & $-\frac{1}{2}$ & 0 & $\frac{1}{2}$ & 0 & $\frac{1}{2}$ & 0 & $(1,1,1,1,1,1)$ & -1 & 0 \\
\hline$D_{+}$ & & $(1,2)$ & 0 & 0 & $-\frac{1}{2}$ & 0 & $-\frac{1}{2}$ & 0 & $-\frac{1}{2}$ & 0 & $(1,1,1,1,1,1)$ & 1 & 0 \\
\hline$D_{-}$ & & $(1,2)$ & 0 & 0 & $\frac{1}{2}$ & 0 & $\frac{1}{2}$ & 0 & $-\frac{1}{2}$ & 0 & $(1,1,1,1,1,1)$ & -1 & 0 \\
\hline$C_{+}^{+-}$ & & $(1,1)$ & 0 & -1 & $\frac{1}{2}$ & 0 & $-\frac{1}{2}$ & 0 & $\frac{1}{2}$ & 0 & $(1,1,1,1,1,1)$ & 1 & 0 \\
\hline$C_{-}^{+-}$ & & $(1,1)$ & 0 & 1 & $\frac{1}{2}$ & 0 & $-\frac{1}{2}$ & 0 & $\frac{1}{2}$ & 0 & $(1,1,1,1,1,1)$ & -1 & 0 \\
\hline$T_{+}$ & $1+b_{4}$ & $(\overline{3}, 1)$ & $-\frac{1}{2}$ & 0 & 0 & $-\frac{1}{2}$ & 0 & 0 & $-\frac{1}{2}$ & 0 & $(1,1,1,1,1,1)$ & 0 & 1 \\
\hline$C_{-}$ & $+\beta$ & $(1,1)$ & $\frac{3}{2}$ & 0 & 0 & $-\frac{1}{2}$ & 0 & 0 & $-\frac{1}{2}$ & 0 & $(1,1$, & 0 & -1 \\
\hline$C_{+}$ & & $(1,1)$ & $-\frac{3}{2}$ & 0 & 0 & $\frac{1}{2}$ & 0 & 0 & $-\frac{1}{2}$ & 0 & $(1,1,1,1,1,1)$ & 0 & 1 \\
\hline$T_{-}$ & & $(3,1)$ & $\frac{1}{2}$ & 0 & 0 & $\frac{1}{2}$ & 0 & 0 & $-\frac{1}{2}$ & 0 & $(1,1,1,1,1,1)$ & 0 & -1 \\
\hline$D_{1}$ & $b_{1}+2 \gamma$ & $(1,1)$ & 0 & 0 & 0 & $-\frac{1}{2}$ & $\frac{1}{2}$ & $-\frac{1}{2}$ & 0 & 0 & & 0 & 0 \\
\hline$S_{1}$ & & $(1$, & 0 & 0 & 0 & $-\frac{1}{2}$ & $\frac{1}{2}$ & $-\frac{1}{2}$ & 0 & 0 & & -1 & -1 \\
\hline$S_{1}^{\prime}$ & & $(1,1)$ & 0 & 0 & 0 & $-\frac{1}{2}$ & $\frac{1}{2}$ & $-\frac{1}{2}$ & 0 & 0 & $(1,1$, & 1 & 1 \\
\hline$\tilde{S}_{1}$ & & $(1,1)$ & 0 & 0 & 0 & $-\frac{1}{2}$ & $\frac{1}{2}$ & $\frac{1}{2}$ & 0 & 0 & $1,1,1)$ & -1 & 1 \\
\hline$\tilde{S}_{1}^{\prime}$ & & $(1,1)$ & 0 & 0 & 0 & $-\frac{1}{2}$ & $\frac{1}{2}$ & $\frac{1}{2}$ & 0 & 0 & $(1,1,1,1,1,1)$ & 1 & -1 \\
\hline$S_{2}$ & $b_{2}+2 \gamma$ & $(1,1$ & 0 & 0 & $-\frac{1}{2}$ & 0 & $\frac{1}{2}$ & 0 & $\frac{1}{2}$ & 0 & $(1,1$ & -1 & 1 \\
\hline$S_{2}^{\prime}$ & & & 0 & 0 & $-\frac{1}{2}$ & 0 & $\frac{1}{2}$ & 0 & $\frac{1}{2}$ & 0 & & 1 & -1 \\
\hline$D_{2}$ & & $(1,1)$ & 0 & 0 & $-\frac{1}{2}$ & 0 & $\frac{1}{2}$ & 0 & $\frac{1}{2}$ & 0 & $(1,1$, & 0 & 0 \\
\hline$\tilde{S}_{2}$ & & $(1,1)$ & 0 & 0 & $-\frac{1}{2}$ & 0 & $\frac{1}{2}$ & 0 & $-\frac{1}{2}$ & 0 & $(1,1,1,1,1,1)$ & -1 & -1 \\
\hline$\tilde{S}_{2}^{\prime}$ & & $(1,1)$ & 0 & 0 & $-\frac{1}{2}$ & 0 & $\frac{1}{2}$ & 0 & $-\frac{1}{2}$ & 0 & $(1,1,1,1,1,1)$ & 1 & 1 \\
\hline$S_{3}$ & $b_{3}+2 \gamma$ & $(1,1)$ & 0 & 0 & $-\frac{1}{2}$ & $-\frac{1}{2}$ & 0 & 0 & 0 & $\frac{1}{2}$ & $(1,1,1,1,1,1)$ & -1 & 1 \\
\hline$S_{3}^{\prime}$ & & $(1,1)$ & 0 & 0 & $-\frac{1}{2}$ & $-\frac{1}{2}$ & 0 & 0 & 0 & $\frac{1}{2}$ & $(1,1$, & 1 & -1 \\
\hline$\tilde{S}_{3}$ & & $(1,1)$ & 0 & 0 & $-\frac{1}{2}$ & $-\frac{1}{2}$ & 0 & 0 & 0 & $-\frac{1}{2}$ & $(1,1,1,1,1,1)$ & -1 & -1 \\
\hline$\tilde{S}_{3}^{\prime}$ & & $(1,1)$ & 0 & 0 & $-\frac{1}{2}$ & $-\frac{1}{2}$ & 0 & 0 & 0 & $-\frac{1}{2}$ & $(1,1,1,1,1,1)$ & 1 & 1 \\
\hline$\tilde{D}_{3}$ & & $(1,1)$ & 0 & 0 & $-\frac{1}{2}$ & $-\frac{1}{2}$ & 0 & 0 & 0 & $-\frac{1}{2}$ & $(1,1,2,1,1,2)$ & 0 & 0 \\
\hline$A_{+}$ & $b_{4}+2 \gamma$ & $(1,1)$ & 0 & 0 & $-\frac{1}{2}$ & 0 & $-\frac{1}{2}$ & 0 & $\frac{1}{2}$ & 0 & $(2,1,1,1,1,1)$ & 0 & 1 \\
\hline$A_{-}$ & & $(1,1)$ & 0 & 0 & & 0 & $\frac{1}{2}$ & 0 & $\frac{1}{2}$ & 0 & $(2,1,1,1,1,1)$ & 0 & -1 \\
\hline
\end{tabular}




\begin{tabular}{|c|c|c|c|c|c|c|c|c|c|c|c|c|c|}
\hline$F$ & SEC & $\begin{array}{c}S U(3) \times \\
S U(2)\end{array}$ & $Q_{C}$ & $Q_{L}$ & $Q_{1}$ & $Q_{2}$ & $Q_{3}$ & $Q_{4}$ & $Q_{5}$ & $Q_{6}$ & $S U(2)_{1, . .6}^{6}$ & $Q_{7}$ & $Q_{8}$ \\
\hline$\tilde{D}_{1}$ & $1+b_{2}+$ & $(1,1)$ & 0 & 0 & 0 & $-\frac{1}{2}$ & $\frac{1}{2}$ & $\frac{1}{2}$ & 0 & 0 & $(1,2,1,2,1,1)$ & 0 & 0 \\
\hline$\tilde{D}_{1}^{\prime}$ & $b_{3}+2 \gamma$ & $(1,1)$ & 0 & 0 & 0 & $-\frac{1}{2}$ & $\frac{1}{2}$ & $\frac{1}{2}$ & 0 & 0 & $(2,1,1,1,2,1)$ & 0 & 0 \\
\hline$\tilde{D}_{2}$ & $1+b_{1}+$ & $(1,1)$ & 0 & 0 & $-\frac{1}{2}$ & 0 & $\frac{1}{2}$ & 0 & $-\frac{1}{2}$ & 0 & $(2,1,1,1,2,1)$ & 0 & 0 \\
\hline \multirow[t]{3}{*}{$\tilde{D}_{2}^{\prime}$} & $b_{3}+2 \gamma$ & $(1,1)$ & 0 & 0 & $-\frac{1}{2}$ & 0 & $\frac{1}{2}$ & 0 & $-\frac{1}{2}$ & 0 & $(1,2,1,2,1,1)$ & 0 & 0 \\
\hline & $1+b_{1}+b_{2}$ & $(1,1)$ & $\frac{3}{4}$ & $\frac{1}{2}$ & $\frac{1}{4}$ & $-\frac{1}{4}$ & $-\frac{1}{4}$ & $\frac{1}{2}$ & 0 & $-\frac{1}{2}$ & $(1,1,1,2,1,1)$ & $\frac{1}{2}$ & $-\frac{1}{2}$ \\
\hline & $b_{4} \pm \gamma$ & $(1,1)$ & $-\frac{3}{4}$ & $-\frac{1}{2}$ & $-\frac{1}{4}$ & $\frac{1}{4}$ & $\frac{1}{4}$ & $-\frac{1}{2}$ & 0 & $-\frac{1}{2}$ & $(1,1,1,2,1,1)$ & $-\frac{1}{2}$ & $\frac{1}{2}$ \\
\hline$\tilde{D}_{3}^{\prime}$ & $1+b_{1}+$ & $(1,1)$ & 0 & 0 & $-\frac{1}{2}$ & $-\frac{1}{2}$ & 0 & 0 & 0 & $-\frac{1}{2}$ & $(2,1,1,1,2,1)$ & 0 & 0 \\
\hline \multirow[t]{5}{*}{$D_{3}$} & $b_{2}+2 \gamma$ & $(1,1)$ & 0 & 0 & $-\frac{1}{2}$ & $-\frac{1}{2}$ & 0 & 0 & 0 & $\frac{1}{2}$ & $(1,2,1,2,1,1)$ & 0 & 0 \\
\hline & $1+b_{1}+$ & $(1,1)$ & 0 & -1 & 0 & 0 & 0 & $-\frac{1}{2}$ & $-\frac{1}{2}$ & $\frac{1}{2}$ & $(2,1,1,1,1,1)$ & 0 & 0 \\
\hline & $b_{2}+b_{3}+$ & $(1,1)$ & 0 & 1 & 0 & 0 & 0 & $\frac{1}{2}$ & $-\frac{1}{2}$ & $\frac{1}{2}$ & $(2,1,1,1,1,1)$ & 0 & 0 \\
\hline & $\beta+2 \gamma$ & $(1,1)$ & 0 & 1 & 0 & 0 & 0 & $-\frac{1}{2}$ & $-\frac{1}{2}$ & $-\frac{1}{2}$ & $(2,1,1,1,1,1)$ & 0 & 0 \\
\hline & & $(1,1)$ & 0 & -1 & 0 & 0 & 0 & $\frac{1}{2}$ & $-\frac{1}{2}$ & $-\frac{1}{2}$ & $(2,1,1,1,1,1)$ & 0 & 0 \\
\hline$D_{-+}^{(3,4)}$ & $1+b_{1}+$ & $(1,1)$ & 0 & 0 & $-\frac{1}{2}$ & 0 & $\frac{1}{2}$ & 0 & $-\frac{1}{2}$ & 0 & $(1,1,2,2,1,1)$ & 0 & 0 \\
\hline$D_{+}^{(5)}$ & $b_{2}+b_{3}$ & $(1,1)$ & 0 & 0 & $-\frac{1}{2}$ & 0 & $-\frac{1}{2}$ & 0 & $-\frac{1}{2}$ & 0 & $(1,1,1,1,2,1)$ & 1 & 0 \\
\hline$D_{-}^{(5)}$ & $\alpha+2 \gamma$ & $(1,1)$ & 0 & 0 & $\frac{1}{2}$ & 0 & $\frac{1}{2}$ & 0 & $-\frac{1}{2}$ & 0 & $(1,1,1,1,2,1)$ & -1 & 0 \\
\hline$D_{+-}^{(3,4)}$ & & $(1,1)$ & 0 & 0 & $\frac{1}{2}$ & 0 & $-\frac{1}{2}$ & 0 & $-\frac{1}{2}$ & 0 & $(1,1,2,2,1,1)$ & 0 & 0 \\
\hline$D_{+-}^{(6)}$ & $\pm \gamma$ & $(1,1)$ & $\frac{3}{4}$ & $\frac{1}{2}$ & $\frac{1}{4}$ & $\frac{1}{4}$ & $\frac{1}{4}$ & $\frac{1}{2}$ & $-\frac{1}{2}$ & 0 & $(1,1,1,1,1,2)$ & $\frac{1}{2}$ & $-\frac{1}{2}$ \\
\hline$D_{--}^{(6)}$ & & $(1,1)$ & $\frac{3}{4}$ & $\frac{1}{2}$ & $\frac{1}{4}$ & $\frac{1}{4}$ & $\frac{1}{4}$ & $-\frac{1}{2}$ & $-\frac{1}{2}$ & 0 & $(1,1,1,1,1,2)$ & $\frac{1}{2}$ & $-\frac{1}{2}$ \\
\hline$D_{++}^{(6)}$ & & $(1,1)$ & $-\frac{3}{4}$ & $-\frac{1}{2}$ & $-\frac{1}{4}$ & $-\frac{1}{4}$ & $-\frac{1}{4}$ & $\frac{1}{2}$ & $-\frac{1}{2}$ & 0 & $(1,1,1,1,1,2)$ & $-\frac{1}{2}$ & $\frac{1}{2}$ \\
\hline$D_{-+}^{(6)}$ & & $(1,1)$ & $-\frac{3}{4}$ & $-\frac{1}{2}$ & $-\frac{1}{4}$ & $-\frac{1}{4}$ & $-\frac{1}{4}$ & $-\frac{1}{2}$ & $-\frac{1}{2}$ & 0 & $(1,1,1,1,1,2)$ & $-\frac{1}{2}$ & $\frac{1}{2}$ \\
\hline$D_{--}^{(3)}$ & $b_{1}+b_{3}$ & $(1,1)$ & $\frac{3}{4}$ & $\frac{1}{2}$ & $-\frac{1}{4}$ & $\frac{1}{4}$ & $-\frac{1}{4}$ & 0 & $-\frac{1}{2}$ & $-\frac{1}{2}$ & $(1,1,2,1,1,1)$ & $-\frac{1}{2}$ & $\frac{1}{2}$ \\
\hline$D_{+-}^{(3)}$ & $\pm \gamma$ & $(1,1)$ & $\frac{3}{4}$ & $\frac{1}{2}$ & $-\frac{1}{4}$ & $\frac{1}{4}$ & $-\frac{1}{4}$ & 0 & $-\frac{1}{2}$ & $\frac{1}{2}$ & $(1,1,2,1,1,1)$ & $-\frac{1}{2}$ & $\frac{1}{2}$ \\
\hline$D_{-+}^{(3)}$ & & $(1,1)$ & $-\frac{3}{4}$ & $-\frac{1}{2}$ & $\frac{1}{4}$ & $-\frac{1}{4}$ & $\frac{1}{4}$ & 0 & $-\frac{1}{2}$ & $-\frac{1}{2}$ & $(1,1,2,1,1,1)$ & $\frac{1}{2}$ & $-\frac{1}{2}$ \\
\hline$D_{++}^{(3)}$ & & $(1,1)$ & $-\frac{3}{4}$ & $-\frac{1}{2}$ & $\frac{1}{4}$ & $-\frac{1}{4}$ & $\frac{1}{4}$ & 0 & $-\frac{1}{2}$ & $\frac{1}{2}$ & $(1,1,2,1,1,1)$ & $\frac{1}{2}$ & $-\frac{1}{2}$ \\
\hline$F$ & $1+b_{3}+\alpha$ & $(1,1)$ & $\frac{3}{4}$ & $-\frac{1}{2}$ & $\frac{1}{4}$ & $-\frac{1}{4}$ & $-\frac{1}{4}$ & $\frac{1}{2}$ & 0 & $\frac{1}{2}$ & $(1,1,2,1,1,1)$ & $-\frac{1}{2}$ & $-\frac{1}{2}$ \\
\hline$F^{\prime}$ & $\beta \pm \gamma$ & $(1,1)$ & $\frac{3}{4}$ & $-\frac{1}{2}$ & $\frac{1}{4}$ & $-\frac{1}{4}$ & $-\frac{1}{4}$ & $\frac{1}{2}$ & 0 & $\frac{1}{2}$ & $(1,1,1,1,1,2)$ & $\frac{1}{2}$ & $\frac{1}{2}$ \\
\hline$\tilde{F}$ & & $(1,1)$ & $-\frac{3}{4}$ & $\frac{1}{2}$ & $-\frac{1}{4}$ & $\frac{1}{4}$ & $\frac{1}{4}$ & $\frac{1}{2}$ & 0 & $-\frac{1}{2}$ & $(1,1,2,1,1,1)$ & $\frac{1}{2}$ & $\frac{1}{2}$ \\
\hline$\tilde{F}^{\prime}$ & & $(1,1)$ & $-\frac{3}{4}$ & $\frac{1}{2}$ & $-\frac{1}{4}$ & $\frac{1}{4}$ & $\frac{1}{4}$ & $\frac{1}{2}$ & 0 & $-\frac{1}{2}$ & $(1,1,1,1,1,2)$ & $-\frac{1}{2}$ & $-\frac{1}{2}$ \\
\hline
\end{tabular}




\begin{tabular}{|c|c|c|rrrrrrrr|r|rr|}
\hline$F$ & SEC & $S U(3) \times$ & $Q_{C}$ & $Q_{L}$ & $Q_{1}$ & $Q_{2}$ & $Q_{3}$ & $Q_{4}$ & $Q_{5}$ & $Q_{6}$ & $S U(2)_{1, \ldots, 6}^{6}$ & $Q_{7}$ & $Q_{8}$ \\
\hline$F_{1}$ & $1+b_{2}+b_{4}$ & $(1,1)$ & $\frac{3}{4}$ & $-\frac{1}{2}$ & $\frac{1}{4}$ & $\frac{1}{4}$ & $\frac{1}{4}$ & $\frac{1}{2}$ & $\frac{1}{2}$ & 0 & $(1,1,2,1,1,1)$ & $-\frac{1}{2}$ & $-\frac{1}{2}$ \\
$F_{2}$ & $\beta \pm \gamma$ & $(1,1)$ & $\frac{3}{4}$ & $-\frac{1}{2}$ & $\frac{1}{4}$ & $\frac{1}{4}$ & $\frac{1}{4}$ & $\frac{1}{2}$ & $-\frac{1}{2}$ & 0 & $(1,1,1,1,1,2)$ & $\frac{1}{2}$ & $\frac{1}{2}$ \\
$F_{3}$ & & $(1,1)$ & $-\frac{3}{4}$ & $\frac{1}{2}$ & $-\frac{1}{4}$ & $-\frac{1}{4}$ & $-\frac{1}{4}$ & $\frac{1}{2}$ & $\frac{1}{2}$ & 0 & $(1,1,2,1,1,1)$ & $\frac{1}{2}$ & $\frac{1}{2}$ \\
$F_{4}$ & & $(1,1)$ & $-\frac{3}{4}$ & $\frac{1}{2}$ & $-\frac{1}{4}$ & $-\frac{1}{4}$ & $-\frac{1}{4}$ & $\frac{1}{2}$ & $-\frac{1}{2}$ & 0 & $(1,1,1,1,1,2)$ & $-\frac{1}{2}$ & $-\frac{1}{2}$ \\
\hline & $1+b_{4}$ & $(1,1)$ & $\frac{3}{4}$ & $\frac{1}{2}$ & $-\frac{1}{4}$ & $\frac{1}{4}$ & $-\frac{1}{4}$ & 0 & $\frac{1}{2}$ & $-\frac{1}{2}$ & $(1,1,1,2,1,1)$ & $\frac{1}{2}$ & $-\frac{1}{2}$ \\
& $\pm \gamma$ & $(1,1)$ & $-\frac{3}{4}$ & $-\frac{1}{2}$ & $\frac{1}{4}$ & $-\frac{1}{4}$ & $\frac{1}{4}$ & 0 & $\frac{1}{2}$ & $-\frac{1}{2}$ & $(1,1,1,2,1,1)$ & $-\frac{1}{2}$ & $\frac{1}{2}$ \\
\hline & $b_{3}+b_{4}$ & $(1,1)$ & $\frac{3}{4}$ & $\frac{1}{2}$ & $\frac{1}{4}$ & $-\frac{1}{4}$ & $-\frac{1}{4}$ & $\frac{1}{2}$ & 0 & $-\frac{1}{2}$ & $(1,2,1,1,1,1)$ & $-\frac{1}{2}$ & $\frac{1}{2}$ \\
& $\pm \gamma$ & $(1,1)$ & $-\frac{3}{4}$ & $-\frac{1}{2}$ & $-\frac{1}{4}$ & $\frac{1}{4}$ & $\frac{1}{4}$ & $-\frac{1}{2}$ & 0 & $-\frac{1}{2}$ & $(1,2,1,1,1,1)$ & $\frac{1}{2}$ & $-\frac{1}{2}$ \\
\hline & $b_{1}+b_{2}+b_{3}$ & $(1,1)$ & $\frac{3}{4}$ & $\frac{1}{2}$ & $-\frac{1}{4}$ & $\frac{1}{4}$ & $-\frac{1}{4}$ & 0 & $-\frac{1}{2}$ & $-\frac{1}{2}$ & $(1,2,1,1,1,1)$ & $-\frac{1}{2}$ & $\frac{1}{2}$ \\
& $+b_{4} \pm \gamma$ & $(1,1)$ & $-\frac{3}{4}$ & $-\frac{1}{2}$ & $\frac{1}{4}$ & $-\frac{1}{4}$ & $\frac{1}{4}$ & 0 & $-\frac{1}{2}$ & $-\frac{1}{2}$ & $(1,2,1,1,1,1)$ & $\frac{1}{2}$ & $-\frac{1}{2}$ \\
\hline
\end{tabular}

